\title{
Relaxation and weak solutions of nonlocal semilinear evolution systems
}

\author{
N. Javaid ${ }^{1 *}$, S. Bilal' ${ }^{1}$, T. Donchev ${ }^{1}$ and A.I. Lazu
}

${ }^{*}$ Correspondence:
nasir.jav7000@gmail.com
${ }^{1}$ Abdus Salam School of
Mathematical Sciences, Lahore,
Pakistan
Full list of author information is
available at the end of the article

\begin{abstract}
We give a relatively short proof of the fact that the solution set of a nonlocal semilinear differential inclusion is dense in the weak solution set of the corresponding convexified system. Moreover, we prove a similar result for the solutions with continuous pseudoderivatives when the right-hand side is continuous with nonempty convex closed and bounded values.
\end{abstract}

MSC: $34 \mathrm{~A} 60$

Keywords: Nonlocal problems; Weak solutions; Density

\section{Introduction}

In this paper we study the following nonautonomous semilinear differential inclusion with nonlocal initial conditions:

$$
\left\{\begin{array}{l}
\dot{x}(t) \in A(t) x(t)+F(t, x(t)), \quad t \in I=[0, S], \\
x(0)=g(x(\cdot))
\end{array}\right.
$$

in a Banach space $E$. Here, $\{A(t) ; t \in I\}$ is a family of densely defined linear operators on $E$, $F: I \times E \rightrightarrows E$ is a multifunction with nonempty closed bounded values, and $g: C(I, E) \rightarrow E$ is a given function.

The nonlocal problems have been intensively studied using various frames and techniques, and they continue to be an important research subject. The interest for this study comes from the fact that these mathematical models describe more accurately than the traditional Cauchy problems the evolution of various phenomena (see, e.g., [5] for details). For results on the existence of mild solutions for nonlocal (autonomous or nonautonomous) semilinear differential equations or inclusions, we mention the papers [2, 13, $15,16,20-22]$. We refer also to [14], where semilinear differential equations with causal operators are studied. For nonlinear nonlocal problems, we mention [1, 3].

In this paper, the first goal is to prove that the solution set of the nonconvex problem (1.1) is dense in the solution set of the so-called relaxed (convexified) problem

$$
\left\{\begin{array}{l}
\dot{x}(t) \in A(t) x(t)+\overline{\mathrm{co}} F(t, x(t)), \\
x(0)=g(x(\cdot)) .
\end{array}\right.
$$

(c) The Author(s) 2019. This article is distributed under the terms of the Creative Commons Attribution 4.0 International License (http://creativecommons.org/licenses/by/4.0/), which permits unrestricted use, distribution, and reproduction in any medium, provided you give appropriate credit to the original author(s) and the source, provide a link to the Creative Commons license, and indicate if changes were made. 
These kinds of results are known in literature as relaxation theorems. These results are very important in the theory of optimal control. The value of the cost functionals associated with system (1.1) does not change under convexification. This problem is studied also in case $A$ is m-dissipative nonlinear. For local problems, where $x(0)=x_{0}$ with $x_{0} \in \overline{D(A)}$, there are many relaxation type theorems for different systems, under various assumptions (see, e.g., $[6,8,10,12,18])$.

In the present paper we prove a relaxation theorem for the nonlocal problem (1.1), assuming that $F(\cdot, \cdot)$ is almost continuous with closed bounded values and $F(t, \cdot)$ is Lipschitz continuous, in general Banach spaces. We should mention that the solution set of (1.2) is not closed in general, even when $A(\cdot)$ generates a compact semigroup (see [7]). In [7] the local autonomous version of (1.1) with $A$ generator of a compact (or equicontinuous) semigroup is studied. More exactly, the notions of limit and weak solutions are defined and the relations between them are studied. In our paper, we introduce these kinds of generalized solutions for our system (1.1) in order to prove our main results. Our hypotheses imply the possibility that the limit solutions may not be mild solutions. We prove that the limit solutions are in fact weak solutions. Further, we prove that the latter have integral representations, and we give a relatively simple proof of the relaxation theorem in general Banach spaces. In a forthcoming paper [4], using a completely different approach, we provide a relaxation theorem in the case when $A$ is an m-dissipative nonlinear operator, but the duality map of $E$ is assumed to be single valued. To our knowledge, these kinds of results are new in the case of nonlocal problems.

The second goal of this paper is to establish the relation between the mild solutions of (1.1) and the solutions of (1.2) with continuous pseudoderivatives. Actually, we consider the case when $F(\cdot, \cdot)$ is jointly continuous with closed convex values, and we prove, using the successive approximations method, that the set of solutions of (1.1) with continuous pseudoderivatives is dense in the set of mild solutions of (1.1).

\section{Preliminaries}

In this section we give the main definitions and notations used along this paper. Moreover, we present the standing hypotheses used throughout the paper.

The Hausdorff metric in the space of the closed bounded subsets of $E$ is $D_{H}(A, B):=$ $\max \{E x(A, B), E x(B, A)\}$, where $E x(A, B)=\sup _{a \in A} \inf _{b \in B}|a-b|$. If $a \in E$ and $B \subset E$, then the distance between $a$ and $B$ is $\operatorname{dist}(a, B)=\inf _{b \in B}|a-b|$. We denote by $\mathbb{B}$ the closed unit ball in $E$. The multifunction $G: I \times E \rightrightarrows E$ is said to be continuous if it is continuous with respect to the Hausdorff metric. It is called almost continuous if for any $\varepsilon>0$ there exists a compact set $I_{\varepsilon} \subset I$ with Lebesgue measure meas $\left(I \backslash I_{\varepsilon}\right)<\varepsilon$ such that $\left.G\right|_{I_{\varepsilon} \times E}$ is continuous. A multifunction $H: I \rightrightarrows E$ is said to be lower semicontinuous (LSC) at $\bar{t}$ if for every $t_{i} \rightarrow \bar{t}$ and every $\bar{h} \in H(\bar{t})$ there exists $h_{i} \in H\left(t_{i}\right)$ such that $h_{i} \rightarrow \bar{h}$. $H$ is called LSC if it is every $t \in I$.

Denote by $L(E)$ the space of all bounded linear operators from the Banach space $E$ into $E$. We recall that a family of bounded linear operators $\{T(t, s) ; 0 \leq s \leq t \leq S\}$ on $E$ is said to be an evolution system if

(i) $T(s, s)=I$ and $T(t, r) T(r, s)=T(t, s)$ for all $0 \leq s \leq r \leq t \leq S$;

(ii) the map $(t, s) \longrightarrow T(t, s)$ is strongly continuous (w.r.t. the operator topology in $L(E)$ ) for $0 \leq s \leq t \leq S$.

Remark 2.1 Due to (ii) there exists $M>0$ such that $M=\sup _{0 \leq s<t \leq S}\|T(t, s)\|_{L(E)}$. 
We refer the reader to [17] for the theory of semilinear equations and evolution operators.

Throughout the paper we consider the following assumption on $A(\cdot)$ :

(A) $\{A(t) ; t \in[0, S]\}$ is a family of closed densely defined unbounded linear operators on $E$ of domains $D(A(t)) \subset E$, with $D(A(t))=D(A)$ not depending on $t$ and dense in $E$, such that there exists an evolution system $\{T(t, s) ; 0 \leq s \leq t \leq S\}$ with the property that $t \longrightarrow T(t, s)$ is differentiable and

$$
\frac{\partial T(t, s)}{\partial t}=A(t) T(t, s) .
$$

Consider the nonautonomous Cauchy problem

$$
\left\{\begin{array}{l}
\dot{x}(t) \in A(t) x(t)+f(t), \quad t \in I, \\
x(0)=x_{0}
\end{array}\right.
$$

where $\{A(t) ; t \in I\}$ satisfies (A), $f(\cdot) \in L^{1}(I, E)$ and $x_{0} \in E$.

Recall that the continuous function $x(\cdot)$ is called the mild solution of (2.1) if it satisfies the integral equation

$$
x(t)=T(t, 0) x_{0}+\int_{0}^{t} T(t, s) f(s) d s
$$

for any $t \in I$. Notice that, for any $0 \leq \tau<t \leq S$, the mild solution $x(\cdot)$ of (2.1) satisfies

$$
x(t)=T(t, \tau) x(\tau)+\int_{\tau}^{t} T(t, s) f(s) d s .
$$

Definition 2.2 A continuous function $x(\cdot)$ is said to be a mild solution of (1.1) if there exists $f(\cdot) \in L^{1}(I, E)$ with $f(t) \in F(t, x(t))$ for a.a. $t \in I$ such that $x(\cdot)$ is a mild solution of (2.1), where $x_{0}$ is replaced by $g(x(\cdot))$.

A function $f(\cdot) \in L^{1}(I, E)$ with $f(t) \in F(t, x(t))$ a.e. on $I$ and satisfying (2.2) will be called a pseudoderivative of $x(\cdot)$. Clearly, the pseudoderivative depends on $x(\cdot)$ and $A(\cdot)$. Since in this paper $A(\cdot)$ is previously defined and does not change, then we will stress only the dependence of the pseudoderivative on the solution.

We end this section by stating the hypotheses on $F$ and $g$.

(F1) $F(\cdot, \cdot)$ is an almost continuous multifunction with nonempty closed bounded values.

(F2) There exists a Lebesgue integrable function $k(\cdot)$ such that $D_{H}(F(t, x), F(t, y)) \leq$ $k(t)|x-y|$ for a.a. $t \in I$ and all $x, y \in E$.

(F3) $\|F(t, 0)\| \leq a(t)$ for any $t \in I$, where $a(\cdot)$ is a Lebesgue integrable function.

(g) $g: C(I, E) \rightarrow E$ is Lipschitz continuous with a constant $\mu>0$, i.e.,

$$
|g(x(\cdot))-g(y(\cdot))| \leq \mu\|x(\cdot)-y(\cdot)\|_{C(I, E)}
$$

for any $x(\cdot), y(\cdot) \in C(I, E)$.

Remark 2.3 It follows from (F2) and (F3) that $\|F(t, x)\| \leq a(t)+k(t)|x|$ for any $(t, x) \in I \times E$. Clearly, (F1) can be relaxed to $F(\cdot, x)$ satisfies Lusin's property. 
Finally, denote $K(t):=\int_{0}^{t} k(s) d s$ and $K:=K(S)$. We need also the following assumption:

(C) $M(\mu+K)<1$.

\section{Main results}

In this section we first study the existence of mild solutions for the nonlocal differential inclusion (1.1). Then, we establish the main result of this section, which is a relaxation theorem. To this end, we use the so-called weak and limit solutions of (1.1).

The following proposition, which is a reformulation of Proposition 2.1 in [1], is used in the proofs of Theorem 3.2 and Lemma 3.7.

Proposition 3.1 Let $G: I \rightrightarrows E$ and $g: I \rightarrow E$ be strongly measurable. Then, for every $\varepsilon>0$, there exists a strongly measurable function $u(\cdot)$ such that $u(t) \in G(t) \cap[g(t)+$ $(\operatorname{dist}(g(t), G(t))+\varepsilon) \mathbb{B}]$ for a.e. $t \in I$.

Theorem 3.2 Under hypotheses (A), (F1), (F2), (F3), (g), and (C), problem (1.1) has a solution.

Proof Take $z_{0} \in E$ and let

$$
y^{0}(t)=T(t, 0) z_{0}+\int_{0}^{t} T(t, s) f_{0}(s) d s, \quad t \in I
$$

where $f_{0}(\cdot)$ is an arbitrary strongly measurable selection of $F\left(\cdot, z_{0}\right)$.

Now we define

$$
y^{1}(t)=T(t, 0) g\left(y^{0}(\cdot)\right)+\int_{0}^{t} T(t, s) f_{1}(s) d s, \quad t \in I,
$$

where $f_{1}(t) \in F\left(t, y^{0}(t)\right)$ a.e. on $I$. Let $\varepsilon_{n}=\frac{1}{2^{n} M S}$ for $n \geq 1$. If $y^{n}(\cdot)$ is known for $n \geq 1$, then we define

$$
y^{n+1}(t)=T(t, 0) g\left(y^{n}(\cdot)\right)+\int_{0}^{t} T(t, s) f_{n+1}(s) d s, \quad t \in I,
$$

where

$$
f_{n+1}(t) \in F\left(t, y^{n}(t)\right) \cap\left(f_{n}(t)+\left(k(t)\left|y^{n}(t)-y^{n-1}(t)\right|+\varepsilon_{n}\right) \mathbb{B}\right) .
$$

Notice that the right-hand side has a strongly measurable selection thanks to Proposition 3.1. In the case when $E$ is separable, one can replace $\varepsilon_{n}$ by 0 . Consequently, for $t \in I$,

$$
\begin{aligned}
\left|y^{n+1}(t)-y^{n}(t)\right| & \leq\left|T(t, 0)\left(g\left(y^{n}(\cdot)\right)-g\left(y^{n-1}(\cdot)\right)\right)\right|+\left|\int_{0}^{t} T(t, s)\left(f_{n+1}(s)-f_{n}(s)\right) d s\right| \\
& \leq M \mu\left\|y^{n}(\cdot)-y^{n-1}(\cdot)\right\|_{C(I, E)}+M \int_{0}^{t}\left(k(s)\left|y^{n}(s)-y^{n-1}(s)\right|+\varepsilon_{n}\right) d s .
\end{aligned}
$$

Thus,

$$
\left\|y^{n+1}(\cdot)-y^{n}(\cdot)\right\|_{C(I, E)} \leq M(\mu+K)\left\|y^{n}(\cdot)-y^{n-1}(\cdot)\right\|_{C(I, E)}+\frac{1}{2^{n}} .
$$


One can prove by induction that

$$
\left\|y^{n+1}(\cdot)-y^{n}(\cdot)\right\|_{C(I, E)} \leq M^{n}(\mu+K)^{n}\left\|y^{1}(\cdot)-y^{0}(\cdot)\right\|+\sum_{i=0}^{n-1}(M(\mu+K))^{i} \frac{1}{2^{n-i}} .
$$

Using $(C)$, we get that $\left(y_{n}(\cdot)\right)_{n}$ is a Cauchy sequence in $C(I, E)$, hence it converges uniformly to some continuous function $y(\cdot)$. As we have chosen the pseudoderivatives, we have that

$$
\left|f_{n+1}(t)-f_{n}(t)\right| \leq k(t)\left|y^{n}(t)-y^{n-1}(t)\right|+\varepsilon_{n}
$$

a.e. on $I$. One can prove that $\left(f_{n}(\cdot)\right)_{n}$ converges strongly in $L^{1}(I, E)$ to $f(\cdot)$. Further, for any $t \in I$,

$$
y(t)=T(t, 0) g(y(\cdot))+\int_{0}^{t} T(t, s) f(s) d s .
$$

Due to (F1) we have $f(t) \in F(t, y(t))$ a.e. on $I$. Therefore, $y(\cdot)$ is a mild solution of (1.1).

Remark 3.3 We note that in the paper [2] the existence of mild solution of (1.1) is proved under similar (but stronger) hypotheses to ours.

Following [7], we define weak solutions and limit solutions of the nonlocal problem (1.1).

Definition 3.4 The continuous function $z(\cdot)$ is said to be a weak solution of $(1.1)$ if $z(0)=$ $g(z(\cdot))$ and

$$
z(t) \in T(t, \tau) z(\tau)+\overline{\int_{\tau}^{t} T(t, s) F(s, z(s)) d s}
$$

for every $0 \leq \tau \leq t \leq S$.

The above integral is in the sense of Aumann.

Definition 3.5 The continuous function $z(\cdot)$ is said to be a limit solution of (1.1) if there exist a sequence of positive numbers $\varepsilon_{n} \downarrow 0$ and a sequence $\left(z_{n}(\cdot)\right)_{n}$ in $C(I, E)$ such that, for every $n, z_{n}(\cdot)$ is a mild solution of

$$
\left\{\begin{array}{l}
\dot{z}_{n}(t) \in A(t) z_{n}(t)+F\left(t, z_{n}(t)+\varepsilon_{n} \mathbb{B}\right), \quad t \in I \\
z_{n}(0)=v_{n}
\end{array}\right.
$$

with $v_{n} \in E$ satisfying $\left|v_{n}-g\left(z_{n}(\cdot)\right)\right|<\varepsilon_{n}$, such that $z(t)=\lim _{n \rightarrow \infty} z_{n}(t)$ uniformly on $I$.

The function $z_{n}(\cdot)$ in (3.1) is called an $\varepsilon_{n}$-solution of (1.1).

Now we state the first main result of this paper.

Theorem 3.6 Under the hypotheses of Theorem 3.2, the solution set of (1.1) is dense in the solution set of the relaxed system (1.2). 
To prove this result we need some preliminary results.

Lemma 3.7 Under the hypotheses of Theorem 3.2, the solution set of (1.1) is dense in the set of limit solutions of (1.1).

Proof Let $y(\cdot)$ be a limit solution of (1.1). Consequently, $y(t)=\lim _{n \rightarrow \infty} z_{n}(t)$, where $\left(z_{n}(\cdot)\right)_{n}$ is a sequence of mild solutions of (3.1) with $\varepsilon_{n} \downarrow 0$. It is easy to see that, in order to get the conclusion, it is enough to show that for every $\delta>0$ there exists $\varepsilon>0$ such that, if $z_{0}(\cdot)$ is an $\varepsilon$-solution of (1.1), then there exists a solution $z(\cdot)$ of (1.1) with $\left\|z(\cdot)-z_{0}(\cdot)\right\|_{C(I, E)}<\delta$.

Let $z_{0}(\cdot)$ be an $\varepsilon$-solution of (1.1) for some $\varepsilon>0$. Using (F2), we have that $\dot{z}_{0}(t) \in$ $A(t) z_{0}(t)+f_{0}(t)+\varepsilon k(t) \mathbb{B}$, where $f_{0}(\cdot) \in L^{1}(I, E)$ with $f_{0}(t) \in F\left(t, z_{0}(t)\right)$ a.e. on $I$, and $\mid z_{0}(0)-$ $g\left(z_{0}(\cdot)\right) \mid<\varepsilon$. Define

$$
z_{1}(t)=T(t, 0) g\left(z_{0}(\cdot)\right)+\int_{0}^{t} T(t, s) f_{0}(s) d s, \quad t \in I .
$$

Then, for every $t \in I$,

$$
\left|z_{1}(t)-z_{0}(t)\right| \leq|T(t, 0)|\left|g\left(z_{0}(\cdot)\right)-z_{0}(0)\right|+\left|\int_{0}^{t} T(t, s) \varepsilon k(s) d s\right| \leq M \varepsilon(K+1) .
$$

Let $f_{1}(\cdot) \in L^{1}(I, E)$ be such that $f_{1}(t) \in F\left(t, z_{1}(t)\right)$ and $\left|f_{1}(t)-f_{0}(t)\right| \leq k(t)\left|z_{1}(t)-z_{0}(t)\right|+\frac{\varepsilon}{2 M S}$ a.e. on I. Define

$$
z_{2}(t)=T(t, 0) g\left(z_{1}(\cdot)\right)+\int_{0}^{t} T(t, s) f_{1}(s) d s, \quad t \in I .
$$

Then, for every $t \in I$,

$$
\begin{aligned}
\left|z_{2}(t)-z_{1}(t)\right| & \leq|T(t, 0)|\left|g\left(z_{1}(\cdot)\right)-g\left(z_{0}(0)\right)\right|+\left|\int_{0}^{t} T(t, s)\right| f_{1}(s)-f_{0}(s)|d s| \\
& \leq M(\mu+K)\left\|z_{1}(\cdot)-z_{0}(\cdot)\right\|_{C(I, E)}+\varepsilon / 2 .
\end{aligned}
$$

We continue successively and, for every $n \geq 1$, define

$$
z_{n+1}(t)=T(t, 0) g\left(z_{n}(\cdot)\right)+\int_{0}^{t} T(t, s) f_{n}(s) d s,
$$

where $f_{n}(t) \in F\left(t, z_{n}(t)\right)$ and

$$
\left|f_{n}(t)-f_{n-1}(t)\right| \leq k(t)\left|z_{n}(t)-z_{n-1}(t)\right|+\frac{\varepsilon}{2^{n} M S}
$$

a.e. on $I$. Thus

$$
\begin{aligned}
\left|z_{n+1}(t)-z_{n}(t)\right| & \leq M\left|g\left(z_{n}(\cdot)\right)-g\left(z_{n-1}(\cdot)\right)\right|+\left|\int_{0}^{t} T(t, s) k(s)\left(z_{n}(s)-z_{n-1}(s)\right) d s\right|+\frac{\varepsilon}{2^{n}} \\
& \leq M(\mu+K)\left\|z_{n}(\cdot)-z_{n-1}(\cdot)\right\|_{C(I, E)}+\frac{\varepsilon}{2^{n}} .
\end{aligned}
$$


Further, we can prove that

$$
\left|z_{n+1}(t)-z_{n}(t)\right| \leq[M(\mu+K)]^{n}\left\|z_{1}(\cdot)-z_{0}(\cdot)\right\|_{C(I, E)}+\varepsilon \sum_{i=0}^{n-1}\left[M(\mu+K)^{i}\right] \frac{1}{2^{n-1}} .
$$

It is easy to see that $\left(z_{n}(\cdot)\right)_{n}$ is Cauchy in $C(I, E)$, since $M(\mu+K)<1$. Therefore, there exists $\lim _{n \rightarrow \infty} z_{n}(t)=z(t)$ uniformly on $I$, where $z(\cdot) \in C(I, E)$. Moreover,

$$
\begin{aligned}
\left|z_{0}(t)-z(t)\right| & \leq \sum_{n=0}^{\infty}[M(\mu+K)]^{n}\left\|z_{1}(\cdot)-z_{0}(\cdot)\right\|_{C(I, E)}+\varepsilon \sum_{n=0}^{\infty} \sum_{i=0}^{n-1}[M(\mu+K)]^{i} \frac{1}{2^{n-i}} \\
& \leq \frac{(M(K+1)+1) \varepsilon}{1-M(\mu+K)}
\end{aligned}
$$

for any $t \in I$.

It remains to show that $z(\cdot)$ is a solution of (1.1). Since $g(\cdot)$ is Lipschitz continuous, we have that $g\left(z_{n}(\cdot)\right) \rightarrow g(z(\cdot))$. By (3.3) we get that $f_{n}(t) \rightarrow f(t)$ strongly in $L^{1}(I, E)$. Then, passing to the limit in (3.2), we obtain

$$
z(t)=T(t, 0) g(z(\cdot))+\int_{0}^{t} T(t, s) f(s) d s
$$

for $t \in I$ and, since $F(\cdot, \cdot)$ is almost continuous, $f(s) \in F(s, z(s))$ a.e. on $I$. Therefore, $z(\cdot)$ is a solution of (1.1).

Lemma 3.8 Under the hypotheses of Theorem 3.2, the set of limit solutions of (1.1) coincides with the set of weak solutions of (1.1). Moreover, both sets are closed.

Proof Let $z(\cdot)$ be a limit solution of (1.1). Then there exist a sequence $\left(\varepsilon_{n}\right)_{n}$ of positive numbers, $\varepsilon_{n} \downarrow 0$, and $\left(z_{n}(\cdot)\right)_{n}$ a sequence of $\varepsilon_{n}$-solutions of (1.1) such that $\lim _{n \rightarrow \infty} z_{n}(t)=$ $z(t)$ uniformly on $I$. Then, for $0 \leq s<t \leq S$,

$$
z_{n}(t)=T(t, s) z_{n}(s)+\int_{s}^{t} T(t, \tau) f_{n}(\tau) d \tau
$$

where $f_{n}(t) \in F\left(t, z_{n}(t)+\varepsilon_{n} \mathbb{B}\right)$ a.e. on $I$ and $\left|z_{n}(0)-g\left(z_{n}(\cdot)\right)\right|<\varepsilon_{n}$. Further, by (F2), we have that

$$
z_{n}(t) \in T(t, s) z_{n}(s)+\int_{s}^{t} T(t, \tau) F\left(\tau, z_{n}(\tau)\right) d \tau+\int_{s}^{t} T(t, \tau) k(\tau) \varepsilon_{n} \mathbb{B} d \tau .
$$

Since $\lim _{n \rightarrow \infty} z_{n}(t)=z(t)$ uniformly on $I$, it follows that $\lim _{n \rightarrow \infty} T(t, s) z_{n}(s)=T(t, s) z(s)$ and since $F(\cdot, \cdot)$ is almost continuous, then, for any $0 \leq s<t \leq S$,

$$
\lim _{n \rightarrow \infty} \int_{s}^{t} T(t, \tau) F\left(\tau, z_{n}(\tau)\right) d \tau=\int_{s}^{t} T(t, \tau) F(\tau, z(\tau)) d \tau .
$$

Consequently, passing to the limit for $n \rightarrow \infty$ in (3.4), we get that

$$
z(t) \in T(t, s) z(s)+\int_{s}^{t} T(t, s) F(\tau, z(\tau)) d \tau
$$


for any $0 \leq s<t \leq S$. Moreover, $\lim _{n \rightarrow \infty} g\left(z_{n}(\cdot)\right)=g(z(\cdot))$, hence $z(0)=g(z(\cdot))$. Therefore, $z(\cdot)$ is a weak solution of (1.1).

Conversely, let $x(\cdot)$ be a weak solution of $(1.1)$, i.e., $x(0)=g(x(\cdot))$ and

$$
x(t) \in T(t, s) x(s)+\overline{\int_{s}^{t} T(t, \tau) F(\tau, x(\tau)) d \tau}
$$

for every $0 \leq s<t \leq S$. Then, using Remark 2.3,

$$
\left.|x(t)| \leq M|x(0)|+M \int_{0}^{t}(a(\tau)+k(\tau)|x(\tau)|) d \tau \leq M\left(|x(0)|+M_{1}\right)+M \int_{0}^{t} k(\tau)|x(\tau)|\right) d \tau,
$$

where $M_{1}=\int_{0}^{S} a(\tau) d \tau$. Consequently, by Gronwall's lemma, we get that $|x(t)| \leq c$ for any $t \in I$, where $c=M\left(|x(0)|+M_{1}\right) \exp (M K)$. Therefore,

$$
\begin{aligned}
|x(t)-x(s)| & \leq|x(t)-T(t, s) x(s)|+|T(t, s) x(s)-x(s)| \\
& \leq M \int_{s}^{t}(a(\tau)+k(\tau) c) d \tau+\|T(t, s)-I\|_{L(E)} c .
\end{aligned}
$$

Then, for every $\varepsilon>0$, there exists a subdivision $0=t_{0}<t_{1}<\cdots<t_{m}<t_{m+1}=S$ such that

$$
|x(t)-x(s)| \leq \frac{\varepsilon}{3}
$$

for any $s, t \in\left[t_{k}, t_{k+1}\right], k \in\{0,1, \ldots, m\}$.

Since

$$
x\left(t_{k+1}\right) \in T\left(t_{k+1}, t_{k}\right) x\left(t_{k}\right)+\overline{\int_{t_{k}}^{t_{k+1}} T\left(t_{k+1}, \tau\right) F(\tau, x(\tau)) d \tau},
$$

then there exists a strongly measurable selection $f_{k}(\tau) \in F(\tau, x(\tau))$, for a.a. $\tau \in\left[t_{k}, t_{k+1}\right)$, such that

$$
x\left(t_{k+1}\right) \in T\left(t_{k+1}, t_{k}\right) x\left(t_{k}\right)+\int_{t_{k}}^{t_{k+1}} T\left(t_{k+1}, \tau\right) f_{k}(\tau) d \tau+\frac{\varepsilon}{3(m+1)\left(M^{m}+1\right)} \mathbb{B} .
$$

Define

$$
z(t)=T(t, 0) g(x(\cdot))+\int_{0}^{t} T(t, s) f(s) d s
$$

where $f(s)=f_{k}(s)$ for $s \in\left[t_{k}, t_{k+1}\right), k \in\{0,1,2, \ldots, m\}$. Notice that

$$
\left|x\left(t_{k+1}\right)-z\left(t_{k+1}\right)\right| \leq \frac{\varepsilon}{3(m+1)\left(M^{m}+1\right)}+M\left|x\left(t_{k}\right)-z\left(t_{k}\right)\right| .
$$

Let $t \in\left[t_{k}, t_{k+1}\right)$ for some $k \in\{0,1,2, \ldots, m\}$. We have that

$$
|x(t)-z(t)| \leq\left|x(t)-x\left(t_{k+1}\right)\right|+\left|x\left(t_{k+1}\right)-z\left(t_{k+1}\right)\right|+\left|z\left(t_{k+1}\right)-z(t)\right| .
$$


Then, due to (3.5), (3.7), one has that $|x(t)-z(t)| \leq \varepsilon$. The latter implies that $f(\cdot)$, the pseudoderivative of $z(\cdot)$, satisfies $f(t) \in F(t, z(t)+\varepsilon \mathbb{B})$ a.e. on $I$. Moreover, $|z(0)-g(z(\cdot))|=$ $|g(x(\cdot))-g(z(\cdot))| \leq \mu\|x(\cdot)-z(\cdot)\| \leq \mu \varepsilon$.

Therefore, $z(\cdot)$ is an $\varepsilon$-solution of (1.1) with $|x(t)-z(t)| \leq \varepsilon$ on $I$. Consequently, $x(\cdot)$ is a limit solution of (1.1).

It remains to show that the set of weak solutions of $(1.1)$ is closed. To this end, let $\left(y^{n}(\cdot)\right)_{n}$ be a sequence of weak solutions of (1.1) with $\lim _{n \rightarrow \infty} y^{n}(t)=y(t)$ uniformly on $I$. Then $\lim _{n \rightarrow \infty} g\left(y^{n}(t)\right)=g(y(t))$ uniformly on $I$. Furthermore, $\lim _{n \rightarrow \infty} F\left(t, y^{n}(t)\right)=F(t, y(t))$ for a.a. $t \in I$, since $F(\cdot, \cdot)$ is almost continuous. Thus, $\lim _{n \rightarrow \infty} T(t, s) F\left(s, y^{n}(s)\right)=T(t, s) F(s, y(s))$ for any $t \in[0, S]$ and a.a. $s \in[0, t]$. It follows that

$$
\lim _{n \rightarrow \infty}\left[T(t, \tau) y^{n}(\tau)+\overline{\int_{\tau}^{t} T(t, s) F\left(s, y^{n}(s)\right) d s}\right]=T(t, \tau) y(\tau)+\overline{\int_{\tau}^{t} T(t, s) F(s, y(s)) d s} .
$$

Therefore,

$$
y(t) \in T(t, \tau) y(\tau)+\overline{\int_{\tau}^{t} T(t, s) F(s, y(s))} d s
$$

for every $0 \leq \tau<t \leq T$.

Lemma 3.9 Under the hypotheses of Theorem 3.2, the set of weak solutions of (1.1) coincides with the set of the weak solutions of the relaxed system (1.2).

Proof Obviously, every weak solution of (1.1) is also a weak solution of the relaxed system (1.2). Let $z(\cdot)$ be a weak solution of (1.2). Then $z(0)=g(z(\cdot))$ and

$$
z(t) \in T(t, s) z(s)+\overline{\int_{s}^{t} T(t, \tau) \overline{\operatorname{co}} F(\tau, z(\tau)) d \tau}
$$

for any $0 \leq s<t \leq S$. Since $T(\cdot, \cdot)$ is a linear evolution operator, one has that $\overline{c o} T(t, s) \times$ $F(s, z(s))=T(t, s) \overline{\mathrm{co}} F(s, z(s))$. Due to the properties of Aumann integral,

$$
\overline{\int_{s}^{t} \overline{\operatorname{co}} T(t, \tau) F(\tau, z(\tau)) d \tau}=\overline{\int_{s}^{t} T(t, \tau) F(\tau, z(\tau)) d \tau} \text {. }
$$

It follows that

$$
z(t) \in T(t, s) z(s)+\overline{\int_{s}^{t} T(t, \tau) F(\tau, z(\tau)) d \tau},
$$

hence $z(\cdot)$ is a weak solution of (1.1).

Proof of Theorem 3.6 We have proved that the solution set of (1.1) is dense in the set of the limit solutions of (1.1) (Lemma 3.7). By Lemmas 3.8 and 3.9, the set of the limit solutions of (1.1) coincides with the set of weak solutions of (1.2). The proof is therefore complete, since the last set contains the solution set of the relaxed system (1.2).

As it was noticed, the solution set of (1.2) is not necessarily closed; however, we can define its closure. 
Remark 3.10 Similar result is proved in a forthcoming paper [4], where the authors study nonlinear system which forces them to assume that the duality map of $E$ is single valued. Here the proof is much shorter due to (in some sense) stronger assumptions. Notice, moreover, that in case of separable $E$, one can replace the almost continuity of $F$ by $F(\cdot, x)$ is measurable for every $x$.

\section{Solutions with continuous pseudoderivatives}

In this section we study the solutions of (1.1) with continuous pseudoderivatives, i.e.,

$$
x(t)=T(t, 0) g(x(\cdot))+\int_{0}^{t} T(t, s) f_{x}(s) d s,
$$

where the function $f_{x}(\cdot)$ is continuous on $I$ and satisfies $f_{x}(s) \in F(s, x(s))$ a.e. on $I$.

We will need the following assumptions on $F(\cdot, \cdot)$, which are stronger than assumptions (F1) and (F2) considered in the previous section.

(F4) $F(\cdot, \cdot)$ is jointly continuous with nonempty closed convex and bounded values.

(F5) There exists a constant $\kappa>0$ such that $D_{H}(F(t, x), F(t, y) \leq \kappa|x-y|$ for every $t \in I$ and every $x, y \in E$.

Remark 4.1 Since $F(\cdot, 0)$ is continuous on $I$, one has that there exists a constant $a>0$ such that $\|F(t, 0)\| \leq a$ for all $t \in I$. Then, by (F5), we have that $\|F(t, x)\| \leq a+\kappa|x|$ for all $t \in I$ and all $x \in E$.

Now we will prove the second relaxation theorem of this paper, which is a particular extension of Theorem 4 from [11].

Theorem 4.2 Assume (A), (F4), (F5), and (g). Moreover, assume that $M(\mu+S \kappa)<1$. Then the set of solutions of (1.1) with continuous pseudoderivatives is nonempty and dense in the set of all solutions of (1.1).

Proof Let $x(\cdot)$ be a mild solution of (1.1) with a pseudoderivative $f_{x}(\cdot) \in L^{1}(I, E)$. Let $G(t)=F(t, x(t))$ for $t \in I$. Then $G$ is continuous with closed convex and bounded values. Furthermore, $\|G(t)\| \leq a+\kappa\left(\max _{t \in I}|x(t)|\right)=\eta$. Thus $\left|f_{x}(t)\right| \leq \eta$ for any $t \in I$.

Let $\varepsilon>0$ and take $\delta \in\left(0, \frac{\varepsilon}{2 M \eta}\right)$. Due to Lusin's theorem, there exists a compact set $I_{\delta} \subset I$ with meas $\left(I \backslash I_{\delta}\right)<\delta$ such that $\left.f_{x}\right|_{I_{\delta}}(\cdot)$ is continuous. Applying now Dugundji extension theorem, we get a continuous function $h_{\delta}(\cdot)$ on $I$ such that $h_{\delta}(t)=f_{x}(t)$ on $I_{\delta}$ and $h_{\delta}(t) \in$ $\operatorname{conv}\left(f_{x}\left(I_{\delta}\right)\right)$ for any $t \in I$. Then $\left|h_{\delta}(t)\right| \leq \eta$ for every $t \in I$. Let

$$
z^{\delta}(t)=T(t, 0) g(x(\cdot))+\int_{0}^{t} T(t, s) h_{\delta}(s) d s
$$

Then

$$
\left|z^{\delta}(t)-x(t)\right| \leq M \int_{I \backslash I_{\delta}}\left|h_{\delta}(s)-f_{x}(s)\right| d s<\varepsilon
$$

for any $t \in I$. 
Let $v>0$. Denote $z^{0}(t):=z^{\delta}(t)$ and $f_{0}(t):=h_{\delta}(t)$ for any $t \in I$. Hence, $\left|z^{0}(t)-x(t)\right|<\varepsilon$ for any $t \in I$ and $f_{0}(t) \in F(t, x(t))$ a.e. on $I$. We define the multifunction $G_{0}: I \rightrightarrows E$ by $G_{0}(t)=f_{0}(t)$ if $x(t)=z^{0}(t)$ and

$$
G_{0}(t)=\overline{\left\{v \in F\left(t, z^{0}(t)\right) ;\left|f_{0}(t)-v\right|<(\kappa+\mu)\left|x(t)-z^{0}(t)\right|\right\}},
$$

otherwise. The multifunction $G_{0}(\cdot)$ is nonempty valued due to (F5). It is easy to see that $G_{0}(\cdot)$ has closed convex values. We claim that $G_{0}(\cdot)$ is LSC. To this end, it is enough to prove that the multifunction $H_{0}: I \rightrightarrows E$ given by $H_{0}(t)=f_{0}(t)$ if $x(t)=z^{0}(t)$ and

$$
H_{0}(t)=\left\{v \in F\left(t, z^{0}(t)\right) ;\left|f_{0}(t)-v\right|<(\kappa+\mu)\left|x(t)-z^{0}(t)\right|\right\}
$$

otherwise, is LSC, since $G_{0}=\overline{H_{0}}$. To this end, let $\bar{t} \in I$ be such that $x(\bar{t}) \neq z^{0}(\bar{t})$. Let $\bar{v} \in H_{0}(\bar{t})$ and the sequence $\left(t_{i}\right)_{i} \subset I$ such that $t_{i} \rightarrow \bar{t}$. Then there exists $\gamma>0$ such that $\left|f_{0}(\bar{t})-\bar{v}\right| \leq$ $(\kappa+\mu)\left|x(\bar{t})-z^{0}(\bar{t})\right|-\gamma$. From (F4), since $z^{0}(\cdot)$ is continuous, we get that the multifunction $F\left(\cdot, z^{0}(\cdot)\right)$ is LSC. Hence, there exists a sequence $\left(v_{i}\right)_{i}$ with $v_{i} \in F\left(t_{i}, z^{0}\left(t_{i}\right)\right)$ such that $v_{i} \rightarrow \bar{v}$. Since the functions $f_{0}(\cdot), x(\cdot)$, and $z^{0}(\cdot)$ are continuous, we get that

$$
\left|f_{0}\left(t_{i}\right)-v_{i}\right|<(\kappa+\mu)\left|x\left(t_{i}\right)-z^{0}\left(t_{i}\right)\right|
$$

for $i$ large enough. Therefore, $v_{i} \in H_{0}\left(t_{i}\right)$ for $i$ large enough, hence $H_{0}(\cdot)$ is LSC in $\bar{t}$. It is easy to see that $H_{0}(\cdot)$ is LSC also in the points $\bar{t} \in I$ with $x(\bar{t})=z^{0}(\bar{t})$.

By Michael's selection theorem (see, e.g., [9, Lemma 2.1]), there exists a continuous selection $f_{1}(t) \in G_{0}(t)$. Hence, $\left|f_{x}(t)-f_{0}(t)\right| \leq(\kappa+v)\left|x(t)-z^{0}(t)\right|$ on $I$. Define

$$
z^{1}(t)=T(t, 0) g\left(z^{0}(\cdot)\right)+\int_{0}^{t} T(t, s) f_{1}(s) d s
$$

for $t \in I$. Then

$$
\begin{aligned}
\left|z^{1}(t)-z^{0}(t)\right| & \leq\|T(t, 0)\|_{L(E)}\left|g(x(\cdot))-g\left(z^{0}(\cdot)\right)\right|+M \int_{0}^{t}\left|f_{0}(s)-f_{1}(s)\right| d s \\
& \leq M \mu\left\|x(\cdot)-z^{0}(\cdot)\right\|_{C(I, E)}+M \int_{0}^{t}(\kappa+v)\left|x(s)-z^{0}(s)\right| d s \\
& \leq M \mu \varepsilon+M S(\kappa+\nu) \varepsilon=M(\mu+S(\kappa+v)) \varepsilon
\end{aligned}
$$

We continue by induction. Let $f_{n}(\cdot)$ and $z^{n}(\cdot)$ be already defined for $n \geq 1$. We define the multifunction $G_{n}: I \rightrightarrows E$ by

$$
G_{n}(t)= \begin{cases}f_{n}(t), & \text { if } z^{n}(t)=z^{n-1}(t), \\ \left\{v \in F\left(t, z^{n}(t)\right) ;\left|f_{n}(t)-v\right|<(\kappa+v)\left|z^{n}(t)-z^{n-1}(t)\right|\right\}, & \text { if } z^{n}(t) \neq z^{n-1}(t) .\end{cases}
$$

We can prove, in the same way as we $\operatorname{did}$ for $G_{0}$, that $G_{n}(\cdot)$ is a nonempty closed convex valued, LSC multifunction. Then there exists a continuous function $f_{n+1}(\cdot)$ such that $f_{n+1}(t) \in G_{n}(t)$ for any $t \in I$. Now, define

$$
z^{n+1}(t)=T(t, 0) g\left(z^{n}(\cdot)\right)+\int_{0}^{t} T(t, s) f_{n+1}(s) d s
$$


for $t \in I$. Then we have that $\left|f_{n+1}(t)-f_{n}(t)\right| \leq(\kappa+v)\left|z^{n}(t)-z^{n-1}(t)\right|$ and

$$
\begin{aligned}
\left|z^{n+1}(t)-z^{n}(t)\right| & \leq M \mu\left\|z^{n}(\cdot)-z^{n-1}(\cdot)\right\|_{C(I, E)}+M S(\kappa+v)\left|z^{n}(t)-z^{n-1}(t)\right| \\
& \leq M(\mu+S(\kappa+v))\left\|z^{n}(\cdot)-z^{n-1}(\cdot)\right\|_{C(I, E)}
\end{aligned}
$$

for any $t \in I$.

Since $M(\mu+S \kappa)<1$, there exists $\bar{v}>0$ such that $M(K+S(\kappa+\nu))<1$ for every $v \leq \bar{v}$.

Consequently, the sequence $\left(z^{n}(\cdot)\right)_{n}$ is Cauchy in $C(I, E)$, hence it converges uniformly to some continuous function $z(\cdot)$. Furthermore, the sequence of continuous functions $\left(f_{n}(\cdot)\right)_{n}$ also converges uniformly to some function $f(\cdot)$, which is also continuous. Moreover,

$$
z(t)=T(t, 0) g(z(\cdot))+\int_{0}^{t} T(t, s) f(s) d s
$$

and $f(t) \in F(t, z(t))$ for any $t \in I$. Finally,

$$
|z(t)-x(t)| \leq \sum_{k=0}^{\infty}\left|z^{k+1}(t)-z^{k}(t)\right|+\left|z^{0}(t)-x(t)\right| \leq \frac{\varepsilon}{1-M(K+S(\kappa+v))} .
$$

That completes the proof since $\varepsilon>0$ is arbitrary.

Remark 4.3 Theorem 4.2 is new even in the case of ordinary differential inclusions in $\mathbb{R}^{n}$.

\section{Example}

Let $\Omega \subset \mathbb{R}^{n}$ be a nonempty open and bounded set with $C^{2}$ boundary $\partial \Omega, \Phi \subset \mathbb{R}^{m}$ be a nonempty bounded open set with smooth boundary $\partial \Phi$, and let $\vec{l} \in \mathbb{R}^{n}$ with $\|\vec{l}\|=\alpha \leq 1$. Consider the following partial differential inclusion:

$$
\begin{aligned}
& \left(\begin{array}{c}
u_{t} \\
v_{t}
\end{array}\right) \in\left(\begin{array}{c}
\alpha \Delta u \\
\left\langle\vec{l}, \nabla_{y} v\right\rangle
\end{array}\right)+G(t, u, v), \quad t \in(0, T), x \in \Omega, y \in \Phi, \\
& \begin{cases}u(t, x)=0 & \text { on }(0, T) \times \partial \Omega, \\
u(0, x)=\beta \int_{\Omega} \int_{0}^{T} h(s, x, \lambda, u(s, \lambda)) d s d \lambda, & x \in \Omega, \\
v(0, y)=\beta v(T, y), & y \in \Phi,\end{cases}
\end{aligned}
$$

where the partial derivatives are taken in the sense of distributions.

Let $X=L^{1}(\Omega)$ and $Y=L^{1}(\Phi)$. Let $E=X \times Y$ with the norm $\|z\|=\max \left\{|u|_{X},|v|_{Y}\right\}$, where $z=(u, v)$ with $u \in X$ and $v \in Y$.

As it is shown in [19, Theorem 7.2.7], the Laplace operator $B$ given by

$$
\left\{\begin{array}{l}
D(B)=\left\{p \in W_{0}^{1,1}(\Omega) ; \Delta p \in X\right\} \\
B p=\Delta p \text { for } p \in D(B)
\end{array}\right.
$$

is linear m-dissipative. Thus, $B$ generates a $C_{0}$-semigroup of contractions $\left\{S_{B}(t) ; t \geq 0\right\}$ (see also [5], p. 38). 
It is well known that the operator $C$ defined by

$$
\left\{\begin{array}{l}
D(C)=\{q \in Y ;\langle\vec{l}, \nabla q\rangle \in Y\}, \\
C(v)=\langle\vec{l}, \nabla v\rangle, \quad \text { for } v \in D(C)
\end{array}\right.
$$

generates an isometry semigroup $\{S(h) ; h \geq 0\}$ given by $(S(h) f)(x)=f(x+\overrightarrow{t l})$ for any $f \in Y$, $h \geq 0, x \in \mathbb{R}^{n}$ (see, e.g., [5, Theorem 1.9.5]).

We assume the following hypotheses.

(G) The multifunction $G$ satisfies the following conditions:

(i) it has nonempty closed values;

(ii) $G(\cdot, u, v)$ is measurable;

(iii) $\|G(\cdot, u, v)\|$ is Lebesgue integrable;

(iv) there exists a Lebesgue integrable function $L(\cdot)$ such that

$$
D_{H}\left(G\left(t, z_{1}\right), G\left(t, z_{2}\right)\right) \leq L(t)\left|z_{1}-z_{2}\right|
$$

$$
\text { for any } t \in[0, T] \text { and any } z_{i}=\left(u_{i}, v_{i}\right) \in \mathbb{R}^{2}, i=1,2 \text {. }
$$

(H) The function $h$ satisfies the conditions:

(i) $h(t, x, \lambda, r)$ is measurable in $(t, x, \lambda)$ for all $r \in \mathbb{R}$;

(ii) there exist a function $H(\cdot) \in C\left(\Omega, \mathbb{R}_{+}\right)$and a positive Lebesgue integrable function $v(\cdot)$ such that $|h(t, x, \lambda, r)| \leq v(t) H(\lambda)$ for any $(t, x, \lambda, r) \in[0, T] \times \Omega \times \Omega \times \mathbb{R}$

(iii) for any $(t, x, \lambda, u),(t, x, \lambda, v) \in[0, T] \times \Omega \times \Omega \times \mathbb{R}$, we have that

$$
|h(t, x, \lambda, u)-h(t, x, \lambda, v)| \leq \frac{K}{T \mu(\Omega)}|u-v| .
$$

Let $\|G(t, 0,0)\| \leq \eta(t)$, where $\eta(\cdot)$ is Lebesgue integrable.

Then we can rewrite problem (5.1)-(5.2) in the abstract form (1.1), where $A=(\alpha B, C)$, the multifunction $F:[0, T] \times E \rightrightarrows E$ is given by

$$
F(t, u(\cdot), v(\cdot))=\left\{\left(z_{1}(\cdot), z_{2}(\cdot)\right) \in E ;\left(z_{1}(x), z_{2}(y)\right) \in G(t, u(x), v(y)) \text { for a.a. } x \in \Omega, y \in \Phi\right\}
$$

for $t \in[0, T], u(\cdot) \in L^{1}(\Omega), v(\cdot) \in L^{1}(\Phi)$, and $g: C([0, T], E) \rightarrow E$,

$$
g\left(w_{1}(\cdot), w_{2}(\cdot)\right)(x, y)=\left(\beta \int_{\Omega} \int_{0}^{T} h\left(s, x, \lambda, w_{1}(s)(\lambda)\right) d s d \lambda, \beta w_{2}(T)(y)\right)
$$

for $w_{1}(\cdot), w_{2}(\cdot) \in C([0, T], E)$.

Let $M=\sqrt{\alpha^{2}+\vec{l}^{2}}$. It is easy to see that all the conditions of Theorem 3.6 then are satisfied if

$$
M\left(\beta+\int_{0}^{T} L(s) d s\right)<1
$$

Applying Theorem 3.6, we obtain the following result. 
Theorem 5.1 Assume (G), (H), and condition (5.3). Then the solution set of (5.1)-(5.2) is nonempty and dense in the solution set of

$$
\left(\begin{array}{c}
u_{t} \\
v_{t}
\end{array}\right) \in\left(\begin{array}{c}
\alpha \Delta u \\
\left\langle\vec{l}, \nabla_{y} v\right\rangle
\end{array}\right)+\overline{c o} G(t, u, v), \quad(t, x, y) \in(0, T) \times \Omega \times \Phi
$$

with

$$
\begin{cases}u(t, x)=0 & \text { оп }(0, T) \times \partial \Omega, \\ u(0, x)=\beta \int_{\Omega} \int_{0}^{T} h(s, x, \lambda, u(s, \lambda)) d s d \lambda, & x \in \Omega, \\ v(0, y)=\beta v(T, y), & y \in \Phi .\end{cases}
$$

\section{Acknowledgements}

NA

\section{Funding}

Not applicable

Availability of data and materials

Please contact the authors for data requests.

\section{Competing interests}

The authors declare that there is no conflict of interests regarding the publication of this paper.

\section{Authors' contributions}

All authors contributed equally and significantly in writing this article. All authors read and approved the final manuscript.

\section{Author details}

'Abdus Salam School of Mathematical Sciences, Lahore, Pakistan. ${ }^{2}$ Department of Mathematics, "Gh. Asachi" Technical University, laşi, Romania.

\section{Publisher's Note}

Springer Nature remains neutral with regard to jurisdictional claims in published maps and institutional affiliations.

Received: 8 January 2019 Accepted: 17 April 2019 Published online: 03 May 2019

\section{References}

1. Ahmed, R., Donchev, T., Lazu, A.I.: Nonlocal m-dissipative evolution inclusions in general Banach spaces. Mediterr. J. Math. 14, 215 (2017). https://doi.org/10.1007/s00009-017-1016-5

2. Aitalionbrahim, M.: Existence of mild solutions of a semilinear nonconvex differential inclusion with nonlocal conditions. Matemat. Vestn. 63, 181-190 (2011)

3. Aizicovici, S., Staicu, V.: Multivalued evolution equations with nonlocal initial conditions in Banach spaces. Nonlinear Differ. Equ. Appl. 14, 361-376 (2007)

4. Bilal, S., Carja, O., Donchev, T., Javaid, N., Lazu, A.l.: Relaxation of nonlocal m-dissipative differential inclusions. An. St. Univ. Ovidius Constanta. To appear

5. Burlică, M., Necula, M., Roşu, D., Vrabie, I.I: Delay Differential Evolutions Subjected to Nonlocal Initial Conditions. Monographs and Research Notes in Mathematics. CRC Press, New York (2016)

6. Cardinali, T., Papageorgiou, N., Papalini, F.: On nonconvex functional evolution inclusions involving m-dissipative operators. Czechoslov. Math. J. 47, 135-148 (1997)

7. Carja, O., Donchev, T., Lazu, A.I.: Generalized solutions of semilinear evolution inclusions. SIAM J. Optim. 26, 1365-1379 (2016)

8. Cârjă, O., Donchev, T., Postolache, V:: Relaxation results for nonlinear evolution inclusions with one-sided Perron right-hand side. Set-Valued Var. Anal. 22(4), 657-671 (2014)

9. Deimling, K.: Multivalued Differential Equations. De Gruyter, Berlin (1992)

10. Donchev, T:: Multi-valued perturbations of m-dissipative differential inclusions in uniformly convex spaces. N.Z. J. Math. 31, 19-32 (2002)

11. Donchev, T., Lazu, A.I., Nosheen, A.: One-sided Perron differential inclusions. Set-Valued Var. Anal. 21, 283-296 (2013)

12. Dong, Y.: Relaxation theorem for evolution differential inclusions. J. Math. Anal. Appl. 237, 188-200 (1999)

13. Fan, Z., Dong, Q., Li, G.: Semilinear differential equations with nonlocal conditions in Banach spaces. Int. J. Nonlinear Sci. 2, 131-139 (2006)

14. Jabeen, T., Lupulescu, V.: Existence of mild solutions of a class of non-autonomous evolution equations with nonlocal initial conditions. J. Nonlinear Sci. Appl. 10, 141-153 (2017) 
15. Li, Y: The global solutions of initial value problem for abstract semilinear evolution equations. Acta Anal. Funct. Appl. 3, 339-347 (2001)

16. Ntouyas, S., Tsamotas, P.: Global existence for semilinear evolution equations with nonlocal conditions. J. Math. Anal. Appl. 210, 679-687 (1997)

17. Pazy, A.: Semigroups of Linear Operators and Applications to Partial Differential Equations. Springer, Berlin (1983)

18. Tolstonogov, A.: Properties of integral solutions of differential inclusions with $\mathrm{m}$-accretive operators. Mat. Zametki 49 , 119-131 (1991)

19. Vrabie, l.: $C_{0}-$ Semigroups and Applications. Elsevier, Amsterdam (2003)

20. Wang, R.N., Zhu, P.X.: Non-autonomous evolution inclusions with nonlocal history conditions: global integral solutions. Nonlinear Anal. 85, 180-191 (2013)

21. Xue, X.: Existence of solutions for semilinear nonlocal Cauchy problems in Banach spaces. Electron. J. Differ. Equ. 2005, 64 (2005)

22. Xue, X: Semilinear nonlocal differential equations with measure of noncompactness in Banach spaces. J. Nanjing. Univ. Math. Big. 24, 264-276 (2007)

Submit your manuscript to a SpringerOpen ${ }^{\circ}$ journal and benefit from:

- Convenient online submission

- Rigorous peer review

Open access: articles freely available online

- High visibility within the field

- Retaining the copyright to your article

Submit your next manuscript at $\boldsymbol{\nabla}$ springeropen.com 